\title{
Analysis and Survey of FPGA Based PROFIBUS
}

\section{Board}

\author{
Nishat Anjum ${ }^{1}$, Sunil Shah ${ }^{2}$ \\ ${ }^{1}$ M.Tech Research Scholar [Embedded System \& VLSI Design], Dept. of ECE, Gyan Ganga Institute of Technology \\ \& Sciences, Jabalpur, Madhya Pradesh, India \\ ${ }^{2}$ Asst. Professor, Dept. of ECE, Gyan Ganga Institute of Technology \& Sciences , Jabalpur, Madhya Pradesh, India
}

\begin{abstract}
Fieldbus system has been established as a communication network, used to connect field devices such as sensors, transduser,controller,man machine interface.Currently a large number of global fieldbus standards are available for consumers.Citation of different types of field bus present an analytical review of various fieldbus systemthat how to gather the data from various equipment distributed far away from each other in the field with reduced wiring and cabling requirement, those are the basic components of communication system. This work also discuss about FPGA based PROFIBUS communication board. It proposes the alternative solution to complexity of communication system and devise it more reliable, high integral and transmission efficient.
\end{abstract}

Keywords-Fieldbus, FPGA, OSI, PROFIBUS, Xilinx.

\section{INTRODUCTION}

Fieldbus is termed to set of network protocol of industrial computer that is applied for real time control. In order to compare different fieldbus system, it is necessary to understand the operation of their predecessor and different communication aspects. This can be evaluated with the OSI reference model that was published in 1984 as the international standard reference model of open systems interconnection (OSI).It is commonly said that fieldbus utilizes only three layers of the OSI model viz. physical layer, data link layer and application layer.

The benefits of the fieldbus approach to inter-device communication in an industrial environment include lower costs, as a result of multiple point to point links that are replaced by a shared bus and the enhancement of the performance of the field device by removing the demand for central computing. The lower cost of fieldbus and the evolution of this technology have led to fieldbus gaining more popularity in communication between any type of controller and controlled devices where point to point links and analogue signals were traditionally used. Table draws a comparison between Fieldbus systems and other networks.

\begin{tabular}{|l|l|l|l|}
\hline S.N & Title & Fieldbus & Other network(LAN) \\
\hline 1 & $\begin{array}{l}\text { Typical } \\
\text { application }\end{array}$ & $\begin{array}{l}\text { Measurement and } \\
\text { control, } \\
\text { automation, } \\
\text { control loops }\end{array}$ & $\begin{array}{l}\text { Office, management, } \\
\text { visualization }\end{array}$ \\
\hline 2 & $\begin{array}{l}\text { Typical type of } \\
\text { station }\end{array}$ & $\begin{array}{l}\text { Sensors, } \\
\text { actuators, devices }\end{array}$ & $\begin{array}{l}\text { Computers, printers, } \\
\text { multi-media }\end{array}$ \\
\hline 3 & $\begin{array}{l}\text { Real-time } \\
\text { requirement }\end{array}$ & Strong & Weak \\
\hline 4 & $\begin{array}{l}\text { Typical user } \\
\text { data }\end{array}$ & $\begin{array}{l}\text { Strings < 100 } \\
\text { bytes }\end{array}$ & Files $>1$ Kbyte \\
\hline 5 & $\begin{array}{l}\text { Typical reaction } \\
\text { time }\end{array}$ & $<1$ second & $\gg 1$ S \\
\hline
\end{tabular}

\section{PROFIBUS}

PROFIBUS is a standard for automation technology. It was first developed in 1989 in Germany. The PROFIBUS Industrial field bus is used to connect controller to remote input and output units, sensors, actuator and inter networking component.PROFIBUS or field bus is an open digital communication system with a wide range of application particularly in the field of factory and process automation. PROFIBUS is suitable for both complex communication taskand time-critical applications.

The application of PROFIBUS include factory automation, drives and motion control, process automation and safety critical application. There are three types of variations used in PROFIBUS. First PROFIBUS DP is used for high speed data communication requiredin factory automation and building automation. Second, PROFIBUS FMS is used for object oriented and general purpose data communication in the cell and field level.Third, PROFIBUS-PAmeets the requirements of the process industry and offers applications for intrinsic safety and non intrinsic safety area and powering the field devices over the bus.

PROFIBUS enhances efficiency of communication system by connecting a number of nodes over a single connection. This single connection not only eliminates the need for dedicated wiring for each node. It also allows reduced complexity, reduced investment and easier 
deployment.PROFIBUS communication technology has multiple protocols such as PROFIBUS DP and PROFIBUS PA that are used depending upon the application requirement. The following features are the reason for choosing PROFIBUS:

- Low comparative cost

- Open standard

- Up to $12 \mathrm{Mbps}$ data transfer rate

- Up to 126 devices per segment

- RS485 physical layer

- Up to 244 bytes of data per transmission

- Hamming distance 4

\section{PROPOSED APPROACH FOR THE PROFIBUS CONTROLLER BOARD}

According to proposed approach PROFIBUS communication controller board will be implemented on Xilinx FPGA. An FPGA is an array of bit processing units whose function and interconnection can be programmed after manufacturing. With the aim of high flexibility, FPGA is chosen to implement the PROFIBUS protocol. The development of a Profibus protocol for FPGA will empower PROFIBUS users and developers to manufacture user specific and cost effective PROFIBUS design. The PROFIBUS design can easily be reprogrammed in order to meet the requirement of a new user or to update the PROFIBUS standard.

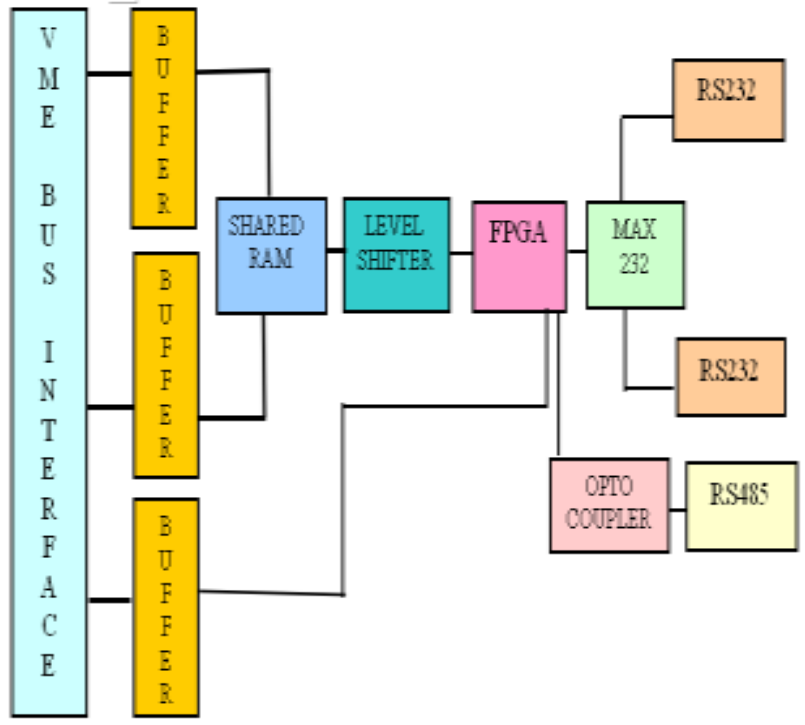

Fig.1: Block diagram of Profibus controller board

The PROFIBUS controller board can be designed in VHDL and implemented on the Xilinx FPGA. The VHDL based programming skills will be established by an introductory course and VHDL literature. The implementation of VHDL based profibus controller board is made by design-entry, synthesis, simulation and programming of FPGA with the Xilinx ISE 12.4 design suit. Impact for loading programming FPGA through JTAG. Once the design meets the PROFIBUS requirement and specification, the design will be extended to meet the requirement of the VME BUS.

The VME BUS specification defines an interfacing system that is used to interconnect the data processing, data storage and peripheral control devices in a closely coupled hardware configuration. The board has been designed with the following objectives

- To allow the communication between VME interfaced devices independent of each other.

- To specify protocol that precisely defines the interaction between the VME bus and devices.

- To provide a system where performance is primarily device limited, rather than system interface limited.

In this paper, it is suggested to implement a PROFIBUS controller board with Master -Slave. It is a bidirectional network meaning that one device, i.e. a master, sends a request to a slave and the slave respond to that request. It supports three layer of ISO/OSI model namely physical layer, data link layer and application layer. In a PROFIBUS communication controller board, physical layerrequired for data transmission andimplemented using FPGA and associated circuitry. Data link layer will beimplemented using FPGA and associated circuitry .It can be configured to be used as a master or a slave.

It provides two types of Services FDL (Field Data Link Layer) andFMA1/2 (Field bus Management layer 1 \&2).Application layer will be implemented on VME Bus compatible 68000/68040 based board running on RTOS OS-9, Which act as FDL and FMA1/2 user.

\section{EXPECTED OUTCOMES FROM THE PROPOSED BOARD}

Through studies and analysis, the hardware and the system proposed, seeking to follow and achieve the aim andis expected to successfully follow and complete the following steps for getting an end module to serve as PROFIBUS communication controller board. These steps areto be generated various modules using VHDL coding then simulation of coding by generating test benches to test.

- Integration of all VHDL programs in a single unit and check the functionality of profibus protocol.

- Hardware development of PROFIBUS Controller Board using FPGA by programming it.

- To implement FDL services tested with 68040 and 68000 based CPU boards as FDL user.

- Porting of the developed PROFIBUS protocol on the board and testing of the hardware circuitry for its full functionalities. 


\section{Required features of proposed board:-}

- VME A24 slave should interface with interrupt generation.

- $16 \mathrm{~Kb}$ SRAM used for data storage.

- $16 \mathrm{~Kb}$ shared RAM, shared by PROFI BUS controller board and VME BUS.

- Possible two UART port:

- One RS232 port with baud rate 9.6k to $19.2 \mathrm{~K}$

- One RS485 port with baud rate 9.6k to 10Mbps.

- On board FPGA and associated circuitry to implement PROFIBUS PROTOCOL for

- Master as well as for Slave mode of operation.

- On board diagnostic and power on self-test facilities.

- Buffering of last few telegrams send and receive.

\section{CONCLUSION AND FUTURE SCOPE}

PROFIBUS Controller board can be used in wide range of applications because of its general properties and it ensures secured data communication as its frame format maintains data integrity.Thedesign of a board will be with flexible, high data integrity, transmission efficiency with simple error detection and provides high data transfer rates. Profibus controller board can serve multiple functions like survey and analysis of several work and respective literature have been done. This survey results in selecting and capturing the approach which serve the desired proposed system.

\section{REFERENCES}

[1] Sheng Liu, Hu Lin,Zhenyu Yinand Meiling Hans, "An Optical Fiber Communication System SupportingMulti-topology for Profibus" Annual IEEE International Conference on Cyber Technology in Automation, Control and Intelligent Systems, 2015.

[2] Xia Lin-lin, Qiu Chao, Fu Zhao-long, Pan Xu-ying“ A Novel Design of Profibus-DP Communication Interface Card for the Intelligent Instruments of Field Network" Proceeding of the IEEE International Conference on Automation and Logistic, 2012.

[3] Panfeng Zhang,Yongzhe Shi,2011 “A Design of Slave Station Interface Circuit of Profibus-DP"9781-4244-9763-8/11/S26.00 @2011 IEEE.

[4] "Developing a decentralized peripheral PROFIBUS corefor a Xilinx FPGA", The School of Electrical, Electronic and Computer Engineering North-West University November 2010.

[5] PROFIBUS Specification Ed - 1.0, March 1998.
[6] F. Xie,H.X. Zhang and J.C. Li, "The Design of PROFIBUS Detecting System Based On MSP430 Microcontroller Slave Station," Control \& Automation,vol. 24, no. 1-2, pp. 105-106, 2008.(in Chinese).

[7] X.H. Li,H.H. Zhang and S.W. Sun, "An intelligent PROFIBUS slave design based on C8051F020 and SPC3," Control \& Automation, vol. 23, no. 9-2, pp. 41-42,59, 2007. (in Chinese)

[8] H.X Sun,T. Liang and L.J. Yun,"Design and Development of Fieldbus Control System Based on PROFIBUS," vol.I, Beijing: National Defense Industry Press,pp.17-50,2007. (in Chinese)

[9] B. Zhu,J.Q. Xia and L.P. Zhou et al, “The Design of PROFIBUS-DP Slave Station Link Layer Controller based on FPGA," Microelectronics \& Computer, vol. 24, no.1, pp. 102-105, 2007. (in Chinese).

[10] L.J.Duan. Fault tree analysis of PROFIBUS-DP Bus Communication in Electric Control System of Oil Drilling Rig[J]. Electric Drive Automation.2010(5): 160-162.

[11] S.Feng. Design and Test of Embedded PROFIBUSDP Slave Station Interface Based on SPC3[J].Computer Technology.2010(10):25182519. 